\title{
Diversity Functions and the Value of Biodiversity
}

\section{Hans-Peter Weikard}

ABSTRACT. Biodiversity loss has been recognized as one of the most important global environmental problems, but the choice of conservation policies is hampered by the lack of an operational concept of biodiversity. Weitzman (1992, 1998) develops a framework for the measurement of diversity and the identification of cost-effective policies for the preservation of biodiversity. Weitzman's framework has been criticized as being unsuitable for the global problem of biodiversity loss. This paper responds to this critique. It is shown that Weitzman's framework of diversity measurement can be made practical and applicable by shifting the level analysis from species to ecosystems. (JEL Q38, D81)

\section{INTRODUCTION}

Biodiversity loss has become a pressing global environmental problem. It may destabilize the ecosystem, it reduces potential sources of food, the chances to discover useful pharmaceuticals, and the aesthetic value of nature. ${ }^{1}$ We obtain very different benefits from nature conservation and it is hard to disentangle the issues involved. As it stands biodiversity is a vague concept and it does not offer much guidance for the design of conservation policies. What is called for is a conceptual framework for the assessment of the value of biodiversity and operational concepts for the assessment of conservation policies. Even if the value of biodiversity cannot actually be measured (e.g., in money terms), because in practice it is hard to make different values commensurable, a conceptual framework would help to clarify the issues and lead us some way toward an operational definition of biodiversity. Seminal work by Martin Weitzman $(1992,1998)$ has prepared the ground. Weitzman (1992) introduces the concept of a diversity function. The fundamental idea is that the diversity of a set of species is a function of the dissimilarity of the species in the set. A diversity function at-

Land Economics • February 2002 • 78 (1): 20-27

ISSN 0023-7639 (C) 2002 by the Board of

Regents of the University of Wisconsin System taches a diversity value to every conceivable set of biological species. Weitzman (1993) suggests the use of genetic information to determine the dissimilarity between species and shows how policy recommendations can be derived for a family of 15 species of cranes. ${ }^{2}$ Given the genetic information, estimates of the survival probabilities and of the marginal costs of "producing" survival probability are sufficient to determine how conservation resources should be allocated to protect genetic diversity in the best possible way. The diversity function approach gives a clear-cut answer to the conservationist's resource allocation problem, if genetic diversity is to be maximized. Of course, the diversity value is only one aspect of the value of conservation. Some species will have value independently of what they contribute to diversity. Weitzman (1998) accounts for that by introducing species value into his framework. Thus, he provides a solution to the "Problem of Noah's Ark": Which species should be taken on board and be protected when the ark is of limited size and not large enough to save all?

While Weitzman's framework seems to be a large step forward toward a rational design of conservation policies, it is not free from problems. Recently, Mainwaring (2001) has raised two important points of criticism. First, a measurement of biodiversity based on

The author is associate professor, Environmental Economics and Natural Resources Group, Department of Social Sciences, Wageningen University, The Netherlands. The author thanks Daniel Bromley, Henk Folmer, Rolf Groeneveld, Ekko van Ierland, David Kleijn, Paul Mensink, and an anonymous referee for helpful comments.

${ }^{1}$ See United Nations Environment Programme (1995) and Perrings et al. (1995) for a detailed account of the various aspects of biodiversity loss; see Randall (1993) for a discussion of philosophical views. Wilson (1992) is a stimulating source for thinking about biodiversity.

${ }^{2}$ Solow, Polasky, and Broadus (1993) have studied the same case using a similar framework. The idea of a diversity measure based on taxonomy has been developed by May (1990) and Vane-Wright, Humphries, and Williams (1991). 
the genetic dissimilarity of species requires more information than will ever be available. Second, even if sufficient genetic information were available, the question remains whether the diversity measure thus obtained captures the relevant values of diversity. While this criticism is correct, it addresses the application of biodiversity measurement on the species level rather than the general framework underlying Weitzman's analysis. This paper shows that information requirements can be met by shifting the level of application from the preservation of species to the preservation of ecosystems while the idea of a diversity function is maintained. A measure of diversity of ecosystems captures the values which drive our concern for maintaining biodiversity.

Gowdy (1997) raises a more fundamental criticism of biodiversity measurement. Gowdy argues that biodiversity is not substitutable. Standard ways of measuring its value are, therefore, not available. Since a functioning ecosystem is essential to the very possibility of human lives, its services cannot be traded for other goods. According to Gowdy $(1997,27)$ we cannot attach a value (be it total or marginal value) to an essential resource. Similarly Mainwaring $(2001,81)$ states that the substitution of ecosystem services is beyond human capacity; examples of non-substitutable services are: "the formation and retention of soils and the maintenance of soil fertility via the nitrogen cycle and the activity of micro-organisms; the breakdown and recycling of organic matter by micro-organisms; provision of fresh water via the hydrological cycle; flood control via flood-plains and wetlands; insect pollination of food crops; pest control via natural predators; and the maintenance of a genetic library" (Mainwaring 2001, 81). Mainwaring accepts to consider the contribution of individual species or individual ecosystems to ecosystem services, but he is doubtful of the results obtained from such exercise. Gowdy $(1997,37)$ takes a more radical view: "Devising a single measure, monetary or otherwise, of the value of biodiversity, is impossible.' However, Gowdy's argument proceeds too quickly. That biodiversity is an essential resource does not imply that any particular species or ecosystem is also essential. The ir- reversibility of species loss is not sufficient for its essentiality.

The claim that we cannot value biodiversity loss is simply false, because we $d o$ value it. A valuation is always implicit in our decisions affecting the survival probabilities of ecosystems and species. By providing a consistent operational framework, diversity theory contributes to a clarification of implicit, hidden, and presumably, inconsistent valuations. In order to implement a rational conservation policy, the value of the set of species surviving under one policy must be compared to the value of the set of species surviving under another. Biodiversity measurement is a necessity and we even have to go beyond this. We do have to weigh biodiversity against other social goals. As long as all essential ecosystem services can be provided, we may well trade ecosystems and species for other goods, as we have done in the past. The issue is rather that biodiversity loss puts essential ecosystem services at risk. Given our limited information and the current pace of biodiversity loss, we cannot be certain that a safe minimum standard is maintained. To be sure, a valuation of essential (i.e., non-substitutable) ecosystem services is impossible, since there are no tradeoffs for the decision-maker. Although, under uncertainty, if there are no safe options, we have to trade off the risk of losing one essential resource against the risk of losing another.

The remainder of the paper is organized as follows. Section 2 discusses whether or not diversity is a relevant source of value in the first place. Section 3 explains the concept of a diversity function. Section 4 introduces the main idea of the paper. In order to render the diversity function approach practical and applicable, it should be concerned with the diversity of ecosystems, not with the diversity of species. Section 5 concludes.

\section{THE VALUE OF (GENETIC) DIVERSITY}

Biodiversity loss concerns different dimensions of value. First, the value of species must be clearly distinguished from the value of diversity. The value of diversity can be de- 
composed into components of instrumental and intrinsic value.

Each species has a particular value: some are sources of food, some offer transport services, others can be used as a source for renewable energy or raw materials for various purposes, and we enjoy the beauty of flowers and butterflies. Biodiversity loss involves a loss of species or, in case of within-species diversity loss, it puts the survival of a species at risk. In any case, one component of the value of biodiversity is species value.

The instrumental value of biodiversity cannot be attributed to particular species. Ecosystem services like the provision of fresh water or the fertility of soils rely essentially on the interaction of different species. Since there is evidence that species diversity on average supports the stability of an ecosystem, diversity as such plays a crucial role in providing these services. ${ }^{3}$ However, we must carefully consider to what unit the concept of diversity should be applied. Genetic diversity seems to be relevant for an assessment of within-species diversity which is a decisive factor for species survival and the potential for development in the evolutionary process. A useful concept of between-species diversity can hardly rely on genetic information. Ecosystem services, for example, are provided by chains of interacting species. What is important, is the functional role of different species in that interaction rather than their genetic makeup. ${ }^{4} \mathrm{~A}$ diverse sample of species does not make a functioning ecosystem. Only for lack of knowledge of the complex interactions in an ecosystem we may focus on maintaining species diversity in order to maintain ecosystem services.

Maintaining diversity also provides an insurance of species value. It is reasonable to rely on a mixed portfolio of species to ensure the availability of food, raw materials, and ecosystem services in an uncertain environment. This value of insurance is the option value of biodiversity. Moreover, not only the availability of particular sources of supply is uncertain but also future demand. The future demand for certain substances, e.g., for pharmaceuticals, can hardly be predicted, since it is generated by the results of future research and our medical and bio-chemical knowl- edge. The possibility of learning gives rise to a quasi-option value of biodiversity. ${ }^{5}$ Like ecosystem services, the option value and the quasi-option value are instrumental diversity values.

The intrinsic value of diversity captures our preference for living in a more diverse and stimulating environment, even if a less diverse environment provides all essential services and sufficient insurance. According to Weitzman $(1993,158)$, "it seems that increasingly many people believe that there is some inherent value in preserving diversity, even though they cannot exactly define what it is." This observation needs some further comment. That increasingly many people attach value to diversity may reflect an increasing awareness of the importance of ecosystem services. Moreover, genetic diversity as such presumably does not mean much to most people. An intrinsic value of diversity is more plausible when it comes to observable features of the different species. We enjoy the diversity of shapes, colors, sounds, and ways of life of the different species. However, the diversity of the observable features does not necessarily correspond to genetic diversity. Genetically similar species may be morphologically quite different and vice versa. The claim that there is an intrinsic value of diversity is too vague. The question that needs to be addressed is: Diversity of what?

\section{THE CONCEPT OF A DIVERSITY FUNCTION}

There are many reasons for biological conservation. The analysis proposed here is based on the assumption that all values can be traced back to a particular attribute of our environment. These can be attributes of a single organism (e.g., the capability to produce a useful substance) or attributes that are incorporated in the interaction of different organisms (e.g., the fertility of soils). ${ }^{6} \mathrm{~A}$ focus

\footnotetext{
${ }^{3}$ McCann (2000) provides a survey of the diversitystability debate.

${ }^{4}$ An example can be found in Mainwaring (2001,

${ }^{5}$ Cf. Bishop and Woodward (1995) and Ready (1995) for surveys.
} 88). 
on the protection of species can be justified only if individuals belonging to the same species have essentially the same attributes. This condition implies that one individual can be replaced by another of the same species while all the relevant attributes are maintained. A concern about biodiversity is a concern about the attributes that are incorporated in an environment with many species. If an environment is rich in terms of biodiversity, it offers more valuable attributes. A poor environment offers less than necessary to survive.

The analysis starts with two observations. First, there seems to be unanimous agreement that biodiversity is desirable. Second, the concern of environmentalists, scientists, and policy makers is to prevent biodiversity loss. It is implicitly acknowledged in this concern that biodiversity is a quantifiable concept. The environment may offer more or less biodiversity, and we can, in principle, compare possible states (of a region, a country, or planet earth) in terms of biodiversity. Given the observations just mentioned, it makes sense to say that the environment under one policy offers more (or less) biodiversity than under another policy. Each policy brings about a certain state of nature and these states can be ordered in terms of biodiversity. Thus, without being committed to a specific content of " biodiversity," we have the following structural framework:

Let $\Omega$ be the set of all possible states of nature and let $\succsim$ be a diversity ordering. Formally, we require that $\succsim$ is a weak ordering on $\Omega .{ }^{7}$ We can now state the following definition:

DEFINITION 1. For all states of nature $\omega$, $\omega^{\prime} \in \Omega, \omega \approx \omega^{\prime}$ if and only if state $\omega$ offers at least as much diversity as state $\omega^{\prime}$.

It is often convenient to represent $\succsim$ by a non-negative real valued function. Such representation is called a diversity function.

DEFINITION 2: A diversity function is a mapping $D: \Omega \rightarrow \Re^{+}$such that for all $\omega, \omega^{\prime} \in$ $\Omega, D(\omega) \geq D\left(\omega^{\prime}\right)$ if and only if $\omega \approx \omega^{\prime}$.
Definitions 1 and 2 are very general. Weitzman's (1992) theory of diversity defines a structure for diversity functions. He explains this theory discussing the example of diversity of species. ${ }^{8}$ A state of nature $\omega$ can be identified with the set of species which exist in this state. Let $\omega_{0}$ be the set of all initially existing species. Weitzman's basic idea is that the diversity of a set of species depends on their dissimilarity. In other words, a measure of diversity of a set of species is an aggregate of the dissimilarity of the species in the set. As a measure of dissimilarity Weitzman $(1992,1993)$ suggests to use taxonomic or genetic information. Such information can be expressed by a distance function which gives the (genetic) distance between any two species in the initial set of species. Formally, a distance function satisfies the following requirements:

DEFINITION 3. A distance function is a mapping $d: \omega_{0} \times \omega_{0} \rightarrow \Re^{+}$such that

$d(x, x)=0$ for all $x \in \omega_{0}$,

$d(x, y)=d(y, x)>0$ for all $x, y \in \omega_{0}$, and $x \neq y$, and $d(x, y) \leq d(x, z)+d(z, y)$ for all $x, y, z \in \omega_{0}$.

The next step of Weitzman's (1992) analysis is to define the dissimilarity $\delta(x, \omega)$ between a species $x$ and a set of species $\omega$. As a measure of dissimilarity, Weitzman uses the standard definition of the distance between a point and a set of points: ${ }^{9}$

$$
\begin{aligned}
& \text { DEFINITION 4. For all } \omega \subset \omega_{0} \text { and all } x \in \omega_{0} \\
& \omega, d(x, \omega)=\min _{y \in \omega} d(x, y)
\end{aligned}
$$

\footnotetext{
${ }^{6}$ Nehring and Puppe (1999) develop a "multi-attribute approach to valuing diversity" which generalizes Weitzman's approach to the measurement of diversity.

${ }^{7} \mathrm{~A}$ weak ordering is a transitive and complete binary relation.

${ }^{8}$ Weitzman (1992, 364) says: "in what follows I shall employ largely biological metaphors." This leaves room for different interpretations and applications. Weitzman (1998, 1279 f) explitcitly states: "In principle, the basic unit could be at the level of the molecule, cell, organ, individual, species, habitat, ecosystem, or so forth.'

${ }^{9} \mathrm{Cf}$. Weikard (1998) for a discussion of dissimilarity as distance.
} 
Finally, Weitzman takes distance $d(x, \omega)$ to measure the diversity difference between a set of species $\omega$ and an enlarged set $\omega \cup\{x\}$, where species $x(x \notin \omega)$ is added to $\omega$. Weitzman's diversity function $D(\omega)$ is recursively defined as follows:

DEFINITION 5. (i) For all $x \in \omega_{0}, D(\{x\})=$ $D_{0}, D_{0} \in \Re^{+}$; and (ii) for all $\omega \subset \omega_{0}$ and all $x \in \omega_{0}, D(\omega \cup\{x\})=D(\omega)+\delta(x, \omega){ }^{10}$

In a later study Weitzman (1993) applies this framework to measure the diversity of a family of 15 species of cranes. If diversity is considered to be valuable, then Weitzman's measurement can serve as a basis for conservation policies. It is important to note, however, that the idea of a diversity function is more general. In general an operational concept of diversity must rely on some measure of dissimilarity between appropriately defined objects. Species diversity is just one special case. The remainder of the paper explores the application of diversity theory to ecosystems.

\section{BIODIVERSITY AS DIVERSITY OF ECOSYSTEMS}

In a recent criticism of Weitzman's approach to diversity measurement Mainwaring $(2001,79)$ stresses the fact that the genetic information necessary to make the approach work will never be available. So far biologists have described no more than 1.75 million of some 13 million species that may exist. ${ }^{11}$ Even for most of the known species we are far from knowing their genetic structure. Since biodiversity loss is an urgent concern and the huge taxonomic task is impossible to complete, the diversity function approach is restricted to very small subsets of species (like the family of cranes, for example). Looking at small subsets, however, cannot solve the problem and may even shift the focus in the wrong direction. An optimal preservation with regard to one small set of species may not be optimal when the biosphere as a whole is at stake. Thus we cannot wait until all the necessary information is gathered, but we also cannot act on partial informa- tion. Mainwaring $(2001,90)$ tentatively suggests that diversity measures can be applied to habitats and ecosystems. ${ }^{12}$ In the remainder of the paper I explore this possibility.

The first step is to give an operational definition of an ecosystem. An ecosystem is:

1. a geographically identifiable region;

2. hosting a set of (interacting) species;

3. and it is viable without (or with limited) biological exchange with other ecosystems.

There are two ways ecosystems can be described using easily observable characteristics: (1) soil and climate conditions; and (2) random samples of species. Presumably two ecosystems with similar conditions will accommodate similar sets of species. Therefore, in order to explain the main ideas of the paper I focus on information of type 2. The aim is to present a measure of diversity of a collection of different ecosystems based on (incomplete) information about the species in each ecosystem.

Along the lines of Section 2 we first define a measure of dissimilarity between two ecosystems. As a measure of dissimilarity serves the number of species contained in one ecosystem and not contained in the other. To state this formally, we use the notation already introduced: $\omega_{0}$ is the set of all existing species. $\Omega$ is the set of all ecosystems $\varepsilon$ (which are subsets of the set of all species). Formally, $\Omega$ is defined to be the set of all non-empty subsets of $\omega_{0}$. Then the formal definition of dissimilarity $\delta\left(\varepsilon, \varepsilon^{\prime}\right)$ between two ecosystems $\varepsilon, \varepsilon^{\prime} \in \Omega$ is as follows:

Definition $3^{\prime}$. For all $\varepsilon, \varepsilon^{\prime} \in \Omega, \delta\left(\varepsilon, \varepsilon^{\prime}\right)=$ $\# \varepsilon-\#\left(\varepsilon \cap \varepsilon^{\prime}\right)$,

where $\# \varepsilon$ denotes the number of species in ecosystem $\varepsilon$ (cf. Weitzman 1998, 1289). ${ }^{13}$

${ }^{10}$ Definition 5 is only adequate for the special case of taxonomic trees. In the general case, part (ii) of Definition 5 must be replaced by $D(\omega)=\max _{\lambda \in \omega}$ $D(\omega \backslash\{x\})+d(x, \omega)$; cf. Weitzman $(1992,375)$.

${ }^{11}$ Mainwaring $(2001,79)$ notes that estimates of the total number of species range between 3.5 million and more than 100 million species.

${ }^{12}$ Weitzman also $(1998,1280)$ mentions this possiblity, but does not pursue it. 
Definition $3^{\prime}$ can be extended in a natural way to define the dissimilarity of an ecosystem and an arbitrary collection of ecosystems $\omega=\left\{\varepsilon_{1}, \varepsilon_{2}, \ldots\right\}, \varepsilon_{i} \in \Omega$. The dissimilarity between ecosystem $\varepsilon$ and a collection of ecosystems $\omega$ is defined as the number of species contained in ecosystem $\varepsilon$ and not contained in any of the ecosystems in the collection $\omega$. This is formally stated as follows:

DEFINITION $4^{\prime}:$ For all $\varepsilon \in \Omega$ and all $\omega \subseteq$
$\Omega, \delta(\varepsilon, \omega)=\# \varepsilon-\#\left(\varepsilon \cap \cup_{\varepsilon_{i} \in \omega} \varepsilon_{i}\right) .{ }^{14}$

This definition of dissimilarity leads to the following diversity function. The diversity of a set of ecosystems $\omega$ is denoted $D(\omega)$; it is recursively defined by:

DEFINITION 5': (i) For all $\varepsilon \in \Omega, D(\{\varepsilon\})=$ $\# \varepsilon$, and (ii) for all $\omega \subseteq \Omega$ and all $\varepsilon \in \Omega$, = $D(\omega \cup\{\varepsilon\})=D(\omega)+\delta(\varepsilon, \omega)$.

\section{The following proposition holds:}

PRoposition 1: The diversity value of a collection of ecosystems $D(\omega)$ is equal to the number of different species in all ecosystems in $\omega$.

Proof: Let $\omega_{n}=\left\{\varepsilon_{1}, \ldots, \varepsilon_{n}\right\}$ be an arbitrary collection of ecosystems. We have to show that for arbitrary $n D\left(\omega_{n}\right)=\# \cup \varepsilon_{i}$. The proof is by induction.

For $n=1$ this holds by part (i) of definition 3: $D\left(\omega_{1}\right)=D\left(\left\{\varepsilon_{1}\right\}\right)=\# \varepsilon_{1}$.

By definitions 2 and $3, D\left(\omega_{n+1}\right)=$ $\# \bigcup_{i=1, \ldots, n} \varepsilon_{i}+\# \varepsilon_{n+1}-\#\left(\varepsilon_{n+1} \cap \bigcup_{i=1, \ldots, n} \varepsilon_{i}\right)$.

Therefore, $D\left(\omega_{n+1}\right)=\# \bigcup_{i=1, \ldots n+1} \varepsilon_{i} \cdot$ Q. QED.

Proposition 1 corroborates the intuitively appealing idea that a comparison of diversity across ecosystems can rely on counting species. By contrast, Weitzman's (1998) measure of diversity of species, that is, diversity in an ecosystem, relies on counting genes. ${ }^{15}$ Proposition 1 tells us that the number of species serves well as a first approximation of diversity given the limited information available. This provides grounding for the intuition that as many species as possible should be saved in order to minimize biodiversity loss. A cost effective conservation policy is a policy which maximizes the number of species subject to a given budget constraint.

The operational framework I have just sketched out is practical and applicable. The biological information input required is the following:

1. A specification of the set of existing ecosystems. The design of a conservation policy must start with an inventory of ecosystems. On a regional level ecosystems like coastal areas, wetlands, various types of agri-ecosystems, and so forth can be identified. Conservation programs on a larger scale must rely on a broader classification, if no inventories exist. For conservation on the global level, a first rough result could be obtained on a low level of precision, using very broadly characterized ecosystems such as the rain forest, Siberia, Australia, or Central European agricultural regions.

2. An estimate of the number of species in each ecosystem.

3. Information about the dissimilarity of ecosystems generated from randomly drawn samples of species from each ecosystem. This information is sufficient to identify the most important ecosystems in terms of diversity value and it gives an indication how to allocate conservation resources. For a full analysis of cost effectiveness of conservation policies additional information is needed.

4. An estimate of protection costs for each ecosystem.

Under uncertainty we cannot fully control whether a species will survive or not and whether an ecosystem will stay intact or not.

\footnotetext{
${ }^{13}$ Note that the dissimilarity relation is not symmetric; only if it is the case that $\# \varepsilon=\# \varepsilon^{\prime}$, then $\delta\left(\varepsilon, \varepsilon^{\prime}\right)=$ $\delta\left(\varepsilon^{\prime}, \varepsilon\right)$.

${ }^{14}$ Definition $4^{\prime}$ is much simpler special case of Weitzman's $(1992,375-84)$ general approach to diversity measurement; see footnote 10 . It also differs from the definition given by Weitzman $(1998,1290)$ where he focuses on taxonomic trees.

${ }^{15}$ Nehring and Puppe (1999) define the diversity of a set of objects as a weighted sum of the relevant attributes of the objects in the set.
} 
Conservation measures affect the probabilities of species extinction and ecosystem loss. Weitzman (1998) derives the expected diversity value on the basis of the assumption of independent survival probabilities for species. This assumption is criticized by Mainwaring (2001, 88-89). The interdependence of the various species is the essence of an ecosystem. The loss of one species may well trigger the loss of additional species. Assuming independent survival probabilities gravely misrepresents a central feature of the ecosystem. This criticism is valid with regard to the diversity of species. If, as I have suggested, diversity theory is applied to ecosystems, the independence of probabilities of ecosystem loss is a plausible assumption, since ecosystems are geographically segregated and ex definitione viable without exchange. ${ }^{16}$ The assumption of independent probabilities is important for a practicable analysis. Even if Weitzman's model would be generalized to account for dependent survival probabilities it would be impossible to empirically estimate the relevant relationships. The informational requirements about the interactions within an ecosystem would be too large.

\section{CONCLUSIONS}

A rational design of conservation policies requires an operational framework, a measurable concept of biodiversity. So far, the discussion has focused on the diversity of species. But for reasons of information constraints this approach does not seem to be promising. Using Weitzman's general framework of diversity measurement, I propose to use ecosystem diversity as a guideline for immediate and urgently required conservation policies. It is shown in the paper that the proposed measure of ecosystem diversity boils down to counting species. A pragmatic conservation policy, thus, aims at maximizing the (expected) number of species under a given budget constraint. Furthermore, it is argued that the protection of ecosystem diversity is broadly in line with the concerns underlying the biodiversity debate: the maintenance of a high species value including in- surance against the loss of species and the maintenance of ecosystem services.

The paper addresses the design of conservation policies assuming a given budget constraint. To determine the size of the budget to be set aside for conservation is a matter that goes beyond the scope of this paper. Indeed, the problem of Noah's ark, which species will be given a place on the ark, is only one of two problems that Noah faces. It is a second-stage problem. At the first stage Noah must make up his mind about how much space on the ark he wants for himself.

\section{REFERENCES}

Bishop, Richard C., and Richard T. Woodward. 1995. "Valuation of Environmental Quality under Certainty." In The Handbook of Environmental Economics, ed. D. W. Bromley. Oxford: Blackwell. 543-67.

Gowdy, John M. 1997. "The Value of Biodiversity: Markets, Society, and Ecosystems." Land Economics 73 (Feb.): 25-41.

Mainwaring, Lynn. 2001. Biodiversity, Biocomplexity, and the Economics of Genetic Dissimilarity. Land Economics 77 (Feb.): 79-93.

McCann, Kevin Shear. 2000. "The DiversityStability Debate.' Nature 405 (May): 228-33.

May, Robert. 1990. "Taxonomy as Destiny. $\mathrm{Na}$ ture 347 (Sept.): 129-30.

Nehring, Klaus, and Clemens Puppe. 1999. "A Theory of Diversity.' Sonderforschungsbereich 303, Discussion Paper A-605, Universität Bonn. 2002. Econometrica forthcoming.

Perrings, Charles, Karl-Göran Mäler, Carl Folke, C. S. Holling, and Bengt-Owe Jansson, eds. 1995. Biodiversity Loss: Economic and Ecological Issues. Cambridge: Cambridge University Press.

Randall, Alan. 1993. "Thinking about the Value of Biodiversity.' Scandinavian Forest Economics 34:4-17.

Ready, Richard C. 1995. "'Environmental Valuation under Uncertainty.' 'In The Handbook of Environmental Economics, 568-93, ed. D. W. Bromley. Oxford: Blackwell.

Solow, Andrew, Stephen Polasky, and James Broadus. 1993. "'On the Measurement of Bio-

\footnotetext{
${ }^{16}$ Migratory species may pose a problem. In this case, the survival of species in one ecosystem might depend on the survival of species in another.
} 
logical Diversity.' Journal of Environmental Economics and Management 24 (1): 60-68.

United Nations Environment Programme. 1995. Global Biodiversity Assessment. Cambridge: Cambridge University Press.

Vane-Wright, R. I., C. J. Humphries, and P. H. Williams. 1991. "What to Protect? - Systematics and the Agony of Choice.' Biological Conservation 55:235-54.

Weikard, Hans-Peter. 1998. "On the Measurement of Diversity.' Institute of Public Economics, Working Paper No. 9801, University of Graz, Austria.
Weitzman, Martin L. 1992. 'OOn Diversity.' Quarterly Journal of Economics. 107 (May): 363-405.

1993. "What to Preserve? An Application of Diversity Theory to Crane Conservation." Quarterly Journal of Economics 108 (Feb.): 157-83.

-. 1998. "The Noah's Ark Problem.' Econometrica 66 (Nov.): 1279-98.

Wilson, Edward O. 1992. The Diversity of Life. Cambridge, Mass: The Belknap Press of Harvard University Press. 